\title{
Association between the gait pattern characteristics of older people and their two-step test scores
}

\author{
Yoshiyuki Kobayashi ${ }^{*}$ (1) and Toru Ogata ${ }^{2}$
}

\begin{abstract}
Background: The Two-Step test is one of three official tests authorized by the Japanese Orthopedic Association to evaluate the risk of locomotive syndrome (a condition of reduced mobility caused by an impairment of the locomotive organs). It has been reported that the Two-Step test score has a good correlation with one's walking ability; however, its association with the gait pattern of older people during normal walking is still unknown. Therefore, this study aims to clarify the associations between the gait patterns of older people observed during normal walking and their Two-Step test scores.

Methods: We analyzed the whole waveforms obtained from the lower-extremity joint angles and joint moments of 26 older people in various stages of locomotive syndrome using principal component analysis (PCA). The PCA was conducted using a $260 \times 2424$ input matrix constructed from the participants' time-normalized pelvic and rightlower-limb-joint angles along three axes (ten trials of 26 participants, 101 time points, 4 angles, 3 axes, and 2 variable types per trial).

Results: The Pearson product-moment correlation coefficient between the scores of the principal component vectors (PCVs) and the scores of the Two-Step test revealed that only one PCV (PCV 2) among the 61 obtained relevant PCVs is significantly related to the score of the Two-Step test.

Conclusions: We therefore concluded that the joint angles and joint moments related to PCV 2-ankle plantarflexion, ankle plantar-flexor moments during the late stance phase, ranges of motion and moments on the hip, knee, and ankle joints in the sagittal plane during the entire stance phase-are the motions associated with the Two-Step test.
\end{abstract}

Keywords: Principal component analysis, Mobility of older people, Two-step test, Gait pattern

\section{Background}

The concept of locomotive syndrome was proposed by the Japanese Orthopedic Association (JOA); it typifies the condition of reduced mobility resulting from a locomotive organ disorder related to aging $[1,2]$. The reduced mobility from musculoskeletal condition, such as knee arthritis, is considered as locomotive syndrome, while the reduced mobility from the neurological condition such as stroke, is not considered as locomotive syndrome. Further, the criteria for locomotive syndrome are

\footnotetext{
* Correspondence: kobayashi-yoshiyuki@aist.go.jp

${ }^{1}$ Digital Human Research Group, Human Informatics Research Institute, National Institute of Advanced Industrial Science and Technology, 2-3-26 Aomi, Koto-ku, Tokyo 135-0064, Japan

Full list of author information is available at the end of the article
}

the same for men and women. Degenerative changes in joints commonly leads to osteoarthritis of the knee and hip, as well as vertebral spondylosis. The dysfunction of the joint and related pain directly impair mobility, and the affected neuronal lesions adjacent to vertebral deformity also cause neurological symptoms. A recent clinical study reported that there are approximately 47 million people in Japan with radiographic knee osteoarthritis, lumber spondylosis, or osteoporosis, indicating they are suffering from, or will be suffering from the locomotive syndrome [3]. The Japanese Ministry of Health, Labor and Welfare reported that females have a higher risk of locomotive syndrome than males [4]. Walking is the most essential means of human mobility.

(c) The Author(s). 2018 Open Access This article is distributed under the terms of the Creative Commons Attribution 4.0 International License (http://creativecommons.org/licenses/by/4.0/), which permits unrestricted use, distribution, and 
Therefore, the detection of the symptoms related to locomotive syndrome and taking corrective measures in the early stages are important to lead and maintain an independent lifestyle.

The JOA prescribed three official tests for assessing the individual risk level of suffering from locomotive syndrome based on the evidence provided in previous studies [5-7] to detect the symptoms of locomotive syndrome in its early stage.: 1) the stand-up test to assess leg strength, 2) the Two-Step test to assess maximal stride length, and 3) a 25-question risk assessment questionnaire to assess the individual's physical conditions and difficulties in daily life. The Two-Step test, which was first developed by Muranaga and Hirano in 2003 [7, 8], intended to assess one's walking ability, including muscle strength, balance, and flexibility of the lower limbs [9]. Previous studies reported that the Two-Step test score was significantly associated with maximal gait speed [6], the risk of falling and degree of independence in daily life [10], gender (the scores of men were significantly higher than those of women) [9], and age (the scores were significantly lower for higher ages in both men and women) [9]. Despite the feasibility of the Two-Step test as a measure of mobility in locomotive syndrome, it is unknown which factors in gait pattern contribute to the score of the test. Revealing those factors, especially for relatively young older people, may provide novel insights for understanding the mechanisms underlying the declining mobility in the early stage of aging. In addition, the characterization of gait patterns of those who score low values in the Two-Step test will lead to the establishment of effective instruction to improve their gait performance in the early stage of locomotive syndrome.

Principal component analysis (PCA) is a technique that has recently attracted much interest in biomechanical studies, because of its usefulness in identifying the movement characteristics of various groups (and under various conditions) using whole data waveforms [11-18]. Nigg et al. [16] have recently pointed out that the success of the traditional gait assessment approach, which investigates a few selected variables at discrete time points, depends on the selection of variables made by the researchers, and can fail to detect potentially interesting results present in large portions of data left unanalyzed. Therefore, although there have been several studies to analyze the association between the Two-Step test score and gait parameters in older people $[6,9,10]$, it is still unclear whether those analyses, in fact, revealed all the dominant components of gait features among the older people. PCA is a multivariate statistical technique that summarizes the information conveyed by many possibly correlated variables using a smaller number of uncorrelated variables (the principal components). PCA generates a set of principal component vectors (PCVs) and one set of principal component scores (PCSs) for each PCV. Each PCV corresponds to one of the orthogonal axes along which the variance of the data is maximal, and each PCS is the projection of the input data onto the corresponding PCV. In PCA, the movements with dominant differences (large variances) appear in the lower-numbered PCVs (and vice versa). Moreover, the data waveforms related to each $\mathrm{PCV}$ can be reconstructed (or simulated) by inputting the voluntary values as PCSs. Thus, if you input +3 and -3 standard deviation values as the PCS of certain PCV(s), exaggerated data waveforms related to the $\mathrm{PCV}(\mathrm{s})$ will be reconstructed, which make it easy to understand the gait features related to the $\mathrm{PCV}(\mathrm{s})$. Therefore, we conclude that PCA can be useful in capturing characteristics of gait patterns and here we attempt to clarify the associations between the gait patterns observed during normal walking and the scores of the Two-Step test.

A previous study [19] focused on the age-related step length reduction and determined that, compared to younger participants, older participants had smaller ankle plantar flexion, lower ankle plantar-flexor moments, and lower ankle plantar-flexor power during the late stance phase. In addition, the older people tended to compensate for these reductions by increasing the hip joint flexor moment and power. We expected a similar decline in the walking ability of older people with low scores on the Two-Step test, when compared to older people with high scores on this test. Therefore, we hypothesized that older people with low scores on the Two-Step test tended to exhibit smaller ankle plantar flexion and lower ankle plantar-flexor moments during the late stance phase, and that they would compensate for these reductions by increasing the hip joint flexor moment.

\section{Methods \\ Participants}

Walking gait data and Two-Step test scores were obtained from 26 older people in various stages of locomotive syndrome ( 9 males and 17 females), aged 60 to 74 . In this study, we recruited relatively young older participants with more women than men, so that we can understand the associations between the gait patterns observed during normal walking and the scores of the Two-Step test in the early stage of aging population. The demographic data of the participants are presented in Table 1. All the participants were capable of walking independently without assistive devices (e.g., canes, crutches, or orthotic devices), had normal or correctedto-normal vision, had no history of neuromuscular disease, and lived independently in their local communities. None of the participants had a history of surgery because of trauma or orthopedic diseases were excluded. Further, we excluded potential participants with any neurological disorder. The experimental protocol was 
Table 1 Average (standard deviation) of the demographic data and spatio-temporal parameters of participants

\begin{tabular}{lllll}
\hline Age $[\mathrm{yrs}]$ & Height $[\mathrm{cm}]$ & Body Mass $[\mathrm{kg}]$ & Step Length $[\mathrm{cm}]$ & Step Width $[\mathrm{cm}]$ \\
$65.2(3.5)$ & $158.2(8.3)$ & $59.0(10.4)$ & $63.7(6.3)$ & $9.4(3.1)$ \\
Stance Time $[\mathrm{s}]$ & Swing Time $[\mathrm{s}]$ & Cadence $[\mathrm{step} / \mathrm{min}]$ & Walking Speed $[\mathrm{m} / \mathrm{s}]$ & \\
$0.58(0.03)$ & $0.40(0.02)$ & $61.65(3.15)$ & $1.32(0.14)$ \\
\hline
\end{tabular}

approved by the local institutional review board (IRB), and all the participants gave their written informed consent before participating.

\section{Measurement}

The Two-Step tests and the gait measurements were performed in a room with a straight 10-m path on which the participants could walk. Each participant was subjected to the Two-Step test prior to gait measurement.

During the Two-Step tests, all participants wore the same type of experimental wear (sleeveless shirt and spats) and shoes provided by the experimenter. The experimental wear and shoe sizes were selected by the participants themselves. Based on the instructions provided by JOA [20], each participant performed the Two-Step test twice, and the best score was recorded.

During gait measurement, the participants were asked to walk barefoot at a comfortable, self-selected speed. Three-dimensional (3D) positional data were obtained during the walk by using reflective markers and a 3D motion capture system (VICON MX, VICON, Oxford, UK) with a $200 \mathrm{~Hz}$ sampling frequency. A total of 57 infrared reflective markers were attached by one of three expert research assistants with more than 10 years of experience, in accordance with the guidelines of the Visual 3D software (C-Motion Inc., Rockville, MD, US). Simultaneously, ground reaction forces (GRFs) were obtained by using seven force plates (BP400600-2000, AMTI, Watertown, MA, US) sampled at $1 \mathrm{kHz}$. Before the walking trials, the positions of the markers were recorded while the participants stood stationary. The participants were then allowed sufficient practice walks to ensure a natural gait. After the practice, ten successful trials were recorded, in which each participant properly stepped on a force plate.

\section{Data analysis}

The raw motion and GRF data were digitally filtered using a zero-lag, fourth-order, low-pass Butterworth filter; the filter cut-off frequencies were $10 \mathrm{~Hz}$ for the positional data and $56 \mathrm{~Hz}$ for the GRF data based on a previous study [21]. The angles of the hip, knee, and ankle joints, and the pelvis-link angle during one gait cycle were calculated for the $\mathrm{x}$-axis (i.e., flexion-extension), y-axis (i.e., abduction-adduction), and $z$-axis (i.e., internal-external rotation) using a Cardan sequence of rotations (X-Y-Z) from the trajectories measured in each trial. The joint moments of the above-mentioned joints on the $x_{-}^{-}, y^{-}$, and $\mathrm{z}$-axis during one gait cycle were calculated from the trajectories and GRFs measured in each trial, using Newton-Euler's inverse-dynamics formula.

The angles and moments were time-normalized by the gait cycle duration determined from the force plate data and divided into 101 variables ranging from 0 to $100 \%$. Therefore, each trial corresponded to a dataset of 2424 variables (101 time points, 4 angles in 3 axes, with 2 types of variables: moment and angle). The step length, step width, stance time, swing time, percentage of the stance-to-swing transition timing, cadence, and walking speed were also determined, to help understand the gait characteristics. The low-pass filtering and all variable calculations (i.e. joint and link angles, joint moments, and spatio-temporal parameters) were performed using the Visual 3D software package.

\section{Statistics}

We applied PCA to the correlation matrix of the 2424 variables calculated from the 260 data points (ten trials for each of the 26 participants); the specific PCA procedure is described in the Appendix. The statistical analyses described below were conducted to identify the associations between the gait patterns observed during normal walking, as represented by the PCVs, and the scores of the Two-Step test. Additionally, for each PCV, simulated joint kinematic and kinetic waveforms were reconstructed from the PCSs with very large or very small values (deviating from the mean by three standard deviations), to interpret the joint angles and joint moments corresponding to the PCVs. These joint kinematic and kinetic waveforms were reconstructed using the technique presented by Kobayashi et al. [13, 14].

To determine the PCVs related to the Two-Step test scores, we calculated the Pearson product-moment correlation coefficient between the Two-Step test scores and the PCSs of the PCVs with contribution rates of 5\% or more. Furthermore, to help understand the gait characteristics related to each PCV, we also calculated the Pearson product-moment correlation coefficient between the PCSs of the PCVs with contribution rates of $5 \%$ or more, the Two-Step test score, and seven different spatio-temporal parameters (step length, step width, stance time, swing time, percentage of the stance-toswing transition timing, cadence, and walking speed). All statistical analyses were executed using the SPSS 
statistical software package (IBM SPSS Statistics Version 23, SPSS Inc., Chicago, IL, USA). Because of the large number of data points $(n=260)$, the correlation coefficients $r$ were considered statistically significant if their values were greater than 0.3, indicating a medium effect size [22].

\section{Results}

The PCA revealed that the first $61 \mathrm{PCVs}$ explained more than $98 \%$ of the total variance. This study focused on the first 19 of these PCVs, each of which explained more than $1 \%$ of the total variance of the 260 gait samples. Together, these first 19 PCVs explained $87.161 \%$ of the variance. The explained variance and the Pearson product-moment correlation coefficients between the PCSs of the first 19 PCVs are shown in Table 2, along with the Two-Step test scores for each PCV. As shown, a significant correlation between the PCVs and the TwoStep test scores was found only on PCV $2(-0.445)$. Figures 1 and 2 show the reconstructed joint kinematic and kinetic waveforms of PCV 2 by presenting the pelvic, hip-joint, knee-joint, and ankle-joint angles and moments along the sagittal, frontal, and horizontal planes; very large (three standard deviation interval) positive (dotted lines) and negative (solid lines) deviations are also illustrated. For the PCV 2, older people with low scores on the Two-Step test tended to exhibit larger PCSs than older people with high scores on the TwoStep test. Therefore, the reconstructed waveforms indicated by the dotted lines in Figs. 1 and 2 (corresponding to very large positive deviations), can be interpreted as representing an exaggerated gait pattern consistent with older people with low scores on the Two-Step test. Similarly, the reconstructed waveforms indicated by the solid lines in Figs. 1 and 2 (corresponding to very large negative deviations), can be interpreted as representing an exaggerated gait pattern consistent with older people with high scores on the Two-Step test. We also provide a stick figure animation of the lower limb movements showing the gaits for PCV 2, to help understand how this PCV affects the joint angles and joint moments (see the Additional file 1).
The Pearson product-moment correlation coefficient revealed that the PCSs of PCV 2 were significantly correlated with the step length $(r=-0.686)$, swing time $(r=-0.375)$, percentage of the stance-to-swing transition timing $(r=-0.318)$, and walking speed $(r=-0.553)$ (Table 3$)$. Further, the scores of the Two-Step test were significantly correlated with the step length $(r=0.319)$, cadence $(r=0.323)$, and walking speed $(r=0.319)$ (Table 3$)$.

\section{Discussion}

The objective of this study was to use PCA to clarify the associations between the gait pattern during normal walking and the Two-Step test scores. We initially hypothesized that older people with low scores on the Two-Step test tended to exhibit smaller ankle plantarflexion and lower ankle plantar-flexor moments during the late stance phase, and that they would compensate for these reductions by increasing the hip joint flexor moment. The results of the present study confirm this hypothesis partially, as described below.

A significant negative correlation between the PCSs and the Two-Step test scores was observed only on PCV 2. In PCA, the movements with dominant differences appear in the lower-numbered PCVs. Therefore, it is reasonable to interpret the motions related to PCV 2which are shown in Figs. 1 and 2-as the gait pattern characteristics associated with the Two-Step test score.

As expected, the reconstructed waveforms (see Figs. 1 and 2) revealed a trend in which older people with low scores on the Two-Step test exhibited smaller ankle plantar-flexion and lower ankle plantar-flexor moments during the late stance phase than older people with high scores on the Two-Step test. These results are similar to the ones of a previous study [19] comparing joint angles and joint moments between healthy young people and older people. However, they did not compensate for these reductions by increasing the hip joint flexor moment. Furthermore, the reconstructed waveforms reveal that older people with low scores on the Two-Step test tend to exhibit a smaller range of motions and moments on the hip, knee, and ankle joints in the sagittal plane

Table 2 Results of main PCA. The main PCA generated a total of 19 PCVs to achieve a cumulate description of $87.161 \%$ of the variability, and only PCV 2 revealed significant correlation with the scores on the two-step test

\begin{tabular}{lllllllllll}
\hline & PCV1 & PCV2 & PCV3 & PCV4 & PCV5 & PCV6 & PCV7 & PCV8 & PCV9 & PCV10 \\
Explained variance (\%) & 18.895 & 9.696 & 9.379 & 7.844 & 5.747 & 4.946 & 4.494 & 3.948 & 3.754 & 2.985 \\
Cumulative (\%) & 18.895 & 28.591 & 37.970 & 45.814 & 51.561 & 56.508 & 61.002 & 64.950 & 68.704 & 71.689 \\
$r$ & -0.250 & -0.445 & 0.010 & 0.252 & 0.083 & 0.149 & -0.033 & 0.134 & -0.053 & -0.116 \\
& PCV11 & PCV12 & PCV13 & PCV14 & PCV15 & PCV16 & PCV17 & PCV18 & PCV19 \\
Explained variance (\%) & 2.550 & 2.378 & 2.132 & 1.786 & 1.753 & 1.385 & 1.280 & 1.185 & 1.025 \\
Cumulative (\%) & 74.238 & 76.616 & 78.748 & 80.534 & 82.287 & 83.672 & 84.952 & 86.136 & 87.161 \\
$r$ & 0.007 & 0.144 & 0.148 & -0.171 & -0.041 & 0.272 & 0.077 & -0.002 & -0.017 \\
\hline
\end{tabular}




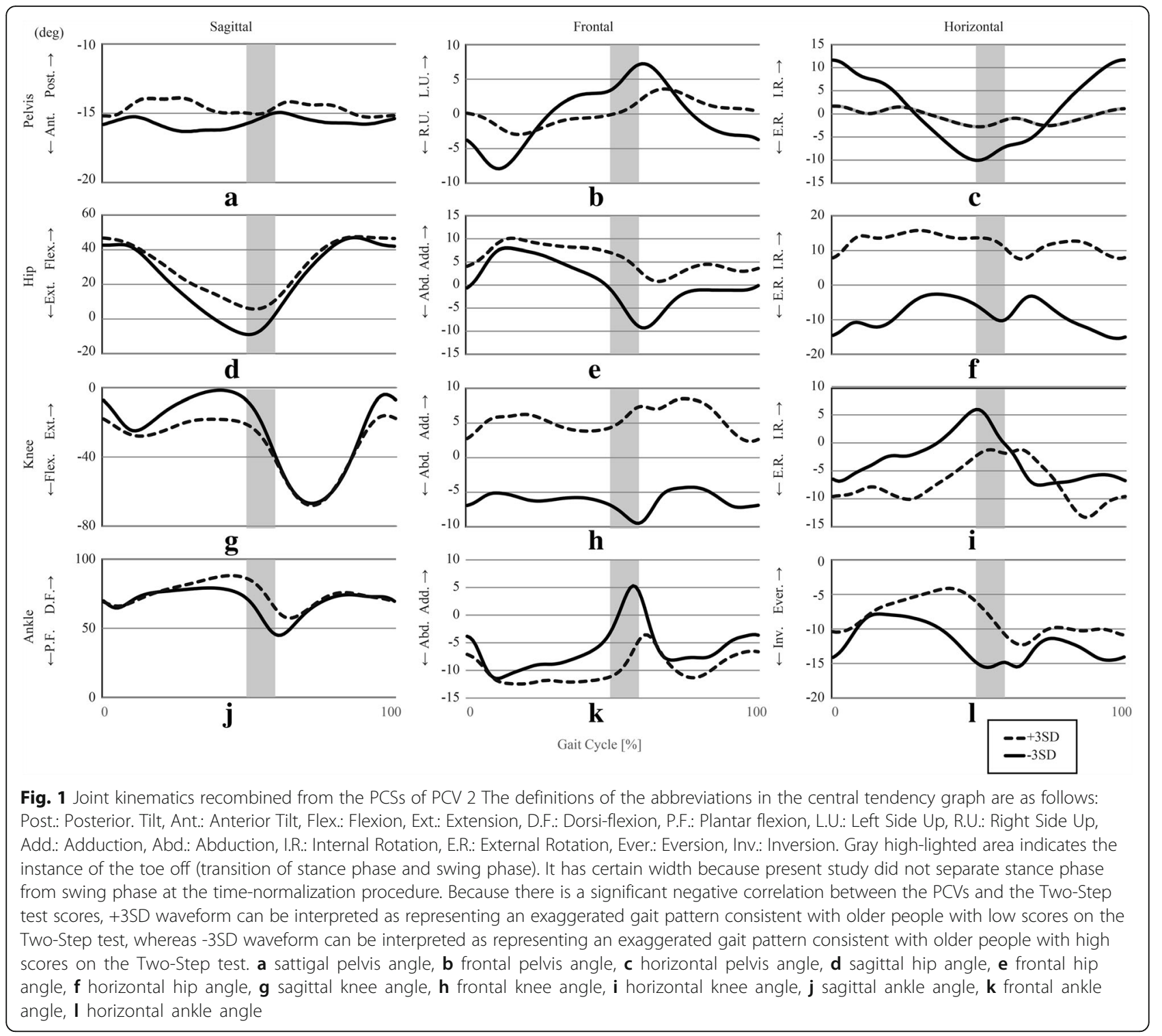

during the entire stance phase, when compared to older people with high scores on the Two-Step test. These results indicate that older people with low scores on the Two-Step test have difficulty maintaining hip motion in the sagittal plane, which is the well-recognized compensation for weak plantar flexion [19], and the Two-Step test can detect such difficulty from the early stage of aging. We assume that this failure in compensating the weak plantar flexion leads to a further reduced ability to progress the body forward during normal walking.

A significant negative correlation was found between the PCSs of PCV 2 and both the swing time $(r=-0.375)$ and the percentage of the stance-to-swing transition timing $(r=-0.318)$. These results indicate that older people with large PCSs on this PCV (i.e., older people with low scores on the Two-Step test) tend to exhibit shorter swing times and smaller percentages of swing phase in one gait cycle than older people with small PCSs on this PCV (i.e., older people with high scores on the Two-Step test). Indeed, some of the figures clearly show a phase shift between the waveforms during the late stance phase. For example, in Fig. 1j, the local minima of the dotted line appear later than those of the solid line.

Most of the traditional gait studies investigated the selected variables only at discrete time points; large portions of the data were therefore not analyzed. PCA, however, can both analyze the whole waveforms and emphasize the gait characteristics of the target populations. These advantages of using PCA enable us to understand and discuss a very comprehensive summary of the gait characteristics associated with the Two-Step test scores. However, there are some factors that must 


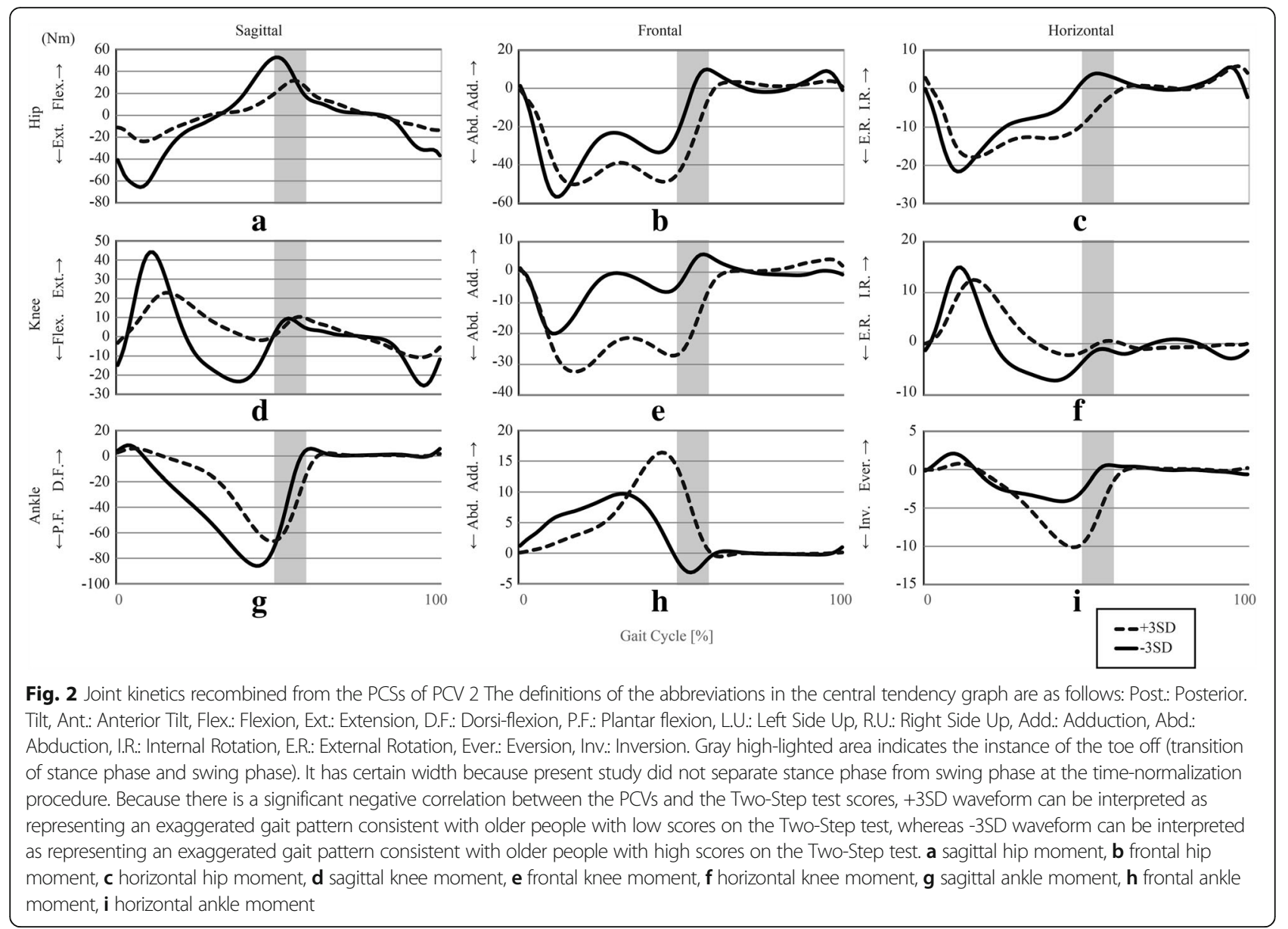

be considered when interpreting the results of the current study. First, we must note that soft tissue artifacts may cause a bias in the observed plane angles, especially in the hip and knee joints. Although we placed markers on the body's bony landmarks, such artifacts must exist. Second, the judgments concerning the significance of correlations were made based on whether the $r$-values were greater than 0.3 , because of the large number of data points $(n=260)$. These limitations may affect the generality of the results of the present study. Therefore, further studies to evaluate and overcome these limitations may still be required. Further, it is noteworthy that participants in this study were relatively young (mean age 65.2 years) and the gait speeds were quite fast $(1.32 \mathrm{~m} / \mathrm{s}$ on average). Therefore, as for clinical implications, the current study shows that the Two-Step test, a newly proposed test for evaluating the presence of the locomotive syndrome, reflects the specific gait pattern and the walking speed even for the relatively young older people. Further accumulation of data and PCA analyses should provide new insights concerning the trajectory of gait deterioration among older people, especially in the case of those with locomotive organ diseases.

\section{Conclusions}

This study used PCA to clarify the associations between the gait patterns during normal walking and the TwoStep test scores. The whole waveforms obtained from the lower-extremity joints' kinematics and kinetics of 26 older people in various stages of the locomotive syndrome were analyzed using PCA. The Pearson productmoment correlation coefficient between the PCSs and the scores of the Two-Step test revealed that only one PCV (PCV 2) among the 61 obtained relevant PCVs was significantly related to the Two-Step test scores.

Table 3 Pearson's product moment correlation between the PCSs of PCV 2 and spatio-temporal parameters

\begin{tabular}{llllllll}
\hline$r$ & Step Length & Step Width & Stance Time & Swing Time & $\%$ of the stance-to-swing transition timing & Cadence & Walking Speed \\
\hline PCV 2 & -0.686 & -0.204 & 0.033 & -0.375 & -0.318 & 0.139 & -0.553 \\
Two-Step test score & 0.319 & -0.049 & -0.067 & 0.234 & -0.043 & 0.323 & 0.319 \\
\hline
\end{tabular}


Therefore, we concluded that the joint angles and joint moments related to PCV 2-ankle plantarflexion, ankle plantar-flexor moments during the late stance phase, range of motions and moments on the hip, knee, and ankle joints in the sagittal plane during the entire stance phase-are the motions associated with the Two-Step test.

\section{Appendix}

PCA procedure adopted in this study.

The PCA analysis conducted as part of this study proceeded as follows. First, we calculated the centered mean of each of the 2424 variables for centering and scaling by using following formula:

$$
z_{\mathrm{tn}}=\left(\mathrm{X}_{\mathrm{tn}}-\mu_{\mathrm{t}}\right) / \sigma_{\mathrm{t}}
$$

where $z_{t n}$ is the scaled data for parameter $t$ and trial $n$, $\mathrm{X}_{\mathrm{tn}}$ is the raw data for parameter $\mathrm{t}$ and trial $\mathrm{n}, \mu_{\mathrm{t}}$ is the mean of parameter $t$ across all trials, and $\sigma_{t}$ is the standard deviation of that same parameter across all trials. The parameter index $t$ ranges between 1 and 2424 and the parameter index $\mathrm{n}$ ranges between 1 and 260 . Second, we constructed a $260 \times 2424$ input matrix containing the ten trials of each of the 26 participants, and their respective 2424 mean-centered parameters. Third, we performed a PCA on the input matrix by using a correlation matrix. Fourth, we conducted the statistical analysis described in the Statistics section above, to identify the PCVs related to the scores of the Two-Step test. Finally, the joint angle and moment waveforms were reconstructed by applying the technique proposed in [9] to interpret the corresponding gait characteristics related to the scores of the Two-Step test.

\section{Additional file}

Additional file 1: Stick figure animation of the lower limb movements relates to PCV 2. This file provides a stick figure animation of the lower limb movements showing the gaits for PCV 2. (GIF 1043 kb)

\section{Abbreviations}

GRF: Ground reaction force; JOA: Japanese Orthopaedic Association; PCA: Principal component analysis; PCV: Principal component vector; PSC: Principal component score

\section{Acknowledgements}

The authors would like to thank all participants, as well as Ms. Yuko Kawai and Ms. Miho Ono for their support on the data acquisition and analysis processes.

\section{Funding}

This study was supported by research aid from the Japanese Orthopaedic Association (JOA-Subsidized Science Project Research 2014-1). This funding source had no role in the design of this study and will not have any role during its execution, analyses, interpretation of the data, or decision to submit results.

\section{Availability of data and materials}

The datasets used and/or analyzed during the current study are available from the corresponding author on reasonable request.

\section{Authors' contributions}

YK designed the experimental setting, performed the experiment, analyzed and interpreted the data, and was a major contributor in writing the manuscript. TO performed the histological examination of the locomotive syndrome, and designed the basic purpose of this study. Both authors read and approved the final manuscript.

\section{Ethics approval and consent to participate}

All procedures performed in studies involving human participants were in accordance with the ethical standards of the institutional and/or national research regulations, and with the 1964 Helsinki declaration and its later amendments or comparable ethical standards.

Written informed consent was obtained from all participants, and all study protocols were approved by the institutional review board of the National Institute of Advanced Industrial Science and Technology (2015-564).

\section{Competing interests}

The authors declare that they have no competing interests.

\section{Publisher's Note}

Springer Nature remains neutral with regard to jurisdictional claims in published maps and institutional affiliations.

\section{Author details}

${ }^{1}$ Digital Human Research Group, Human Informatics Research Institute, National Institute of Advanced Industrial Science and Technology, 2-3-26 Aomi, Koto-ku, Tokyo 135-0064, Japan. ${ }^{2}$ National Rehabilitation Center for Persons with Disabilities, 4-1 Namiki, Tokorozawa-shi, Saitama, Japan.

Received: 18 August 2017 Accepted: 5 April 2018

Published online: 27 April 2018

References

1. Nakamura K. A "super-aged" society and the "locomotive syndrome". J Orthop Sci. 2008;13:1-2.

2. Iwaya T, Doi T, Seichi A, Hoshino Y, Ogata T, Akai M. Characteristics of disability in activity of daily living in elderly people associated with locomotive disorders. BMC Geriatr. 2017;17:165.

3. Yoshimura N, Muraki S, Oka H, et al. Prevalence of knee osteoarthritis, lumbar spondylosis, and osteoporosis in Japanese men and women: the research on osteoarthritis/osteoporosis against disability study. J Bone Miner Metab. 2009;27(5):620-8.

4. Household Statistics Office, Ministry of Health, Labor and Welfare, Summary of Comprehensive Survey of Living Conditions, 2004. http://www.mhlw.go. jp/english/database/db-hss/cslc-index.html. Accessed 12 Apr 2018.

5. Hoshino Y, Seichi A, Iwaya T, Akai M, Tobimatsu Y, Doi T. Development of diagnostic-tool for locomotive syndrome. J Jpn Orthop Assoc. 2011;85:12-20.

6. Muranaga S. Evaluation of the muscular strength of the lower extremities using the standing movement and clinical application. J Showa Med Assoc. 2001;61(3):362-7.

7. Muranaga S, Hirano K. Development of a convenient way to predict ability to walk, using a two-step test. J Showa Med Assoc. 2003;63(3):301-8.

8. Japanese Orthopaedic Association. Locomotive Syndrome Pamphlet 2013.

9. Yoshimura N, Muraki S, Oka H, Tanaka S, Ogata T, Kawaguchi H, Akune T, Nakamura K. Association between new indices in the locomotive syndrome risk test and decline in mobility: third survey of the ROAD study. J Orthop Sci. 2015;20(5):896-905.

10. Yoshimura N, Muraki S, Oka H, Kawaguchi H, Nakamura K, Akune T. Cohort profile: research on osteoarthritis/osteoporosis against disability (road) study. Int J Epidemiol. 2010;39(4):988-95.

11. Deluzio KJ, Astephen JL. Biomechanical features of gait waveform data associated with knee osteoarthritis: an application of principal component analysis. Gait \& posture. 2007;25(1):86-93.

12. Federolf PA, Boyer KA, Andriacchi TP. Application of principal component analysis in clinical gait research: identification of systematic differences between healthy and medial knee-osteoarthritic gait. J Biomech. 2013; 46(13):2173-8. 
13. Kobayashi Y, Hobara H, Matsushita S, Mochimaru M. Key joint kinematic characteristics of the gait of fallers identified by principal component analysis. J Biomech. 2014;47(10):2424-9.

14. Kobayashi Y, Hobara H, Heldoorn TA, Kouchi M, Mochimaru M. Ageindependent and age-dependent sex differences in gait pattern determined by principal component analysis. Gait \& posture. 2016;46:11-7.

15. Maurer C, Federolf P, von Tscharner V, Stirling L, Nigg BM. Discimination of gender-, speed-, and shoe-dependent movement patterns in runners using full-body kinematics. Gait \& posture. 2012;36(1):40-5.

16. Nigg BM, Baltich J, Maurer C, Federolf P. Shoe midsole hardness, sex and age effects on lower extremity kinematics during running. J Biomech. 2012;45(9):1692-7.

17. Raptopoulos LS, Dutra MS, Pinto FA, Pina FA. Alternative approach to modal gait analysis through the Karhunen-Loeve decomposition: an application in the sagittal plane. J Biomech. 2006;39(15):2898-906.

18. Wrigley AT, Albert WJ, Deluzio KJ, Stevenson JM. Differentiating lifting technique between those who develop low back pain and those who do not. Clin Biomech (Bristol, Avon). 2005;20(3):254-63.

19. Judge JO, Davis RB III, Õunpuu S. Step length reductions in advanced age: the role of ankle and hip kinetics. J Gerontol A Biol Sci Med Sci. 1996;51(6) M303-12.

20. Japanese Orthopaedic Association. Locomotive Syndrome Pamphlet 2015. https://locomo-joa.jp/en/index.pdf. Accessed 16 Aug 2017.

21. van den Bogert AJ and de Koning JJ. On onptimal filtering for inverse dynamics analysis. Proceedings of the IXth Biennial Conference of the Canadian Society for Biomechanics, 1996, pp. 214-215.

22. Cohen J. The analysis of variance. Statistical power analysis for the behavioral sciences. New Jersey: Lawrence Erlbaum Associates, Publishers; 1988. p. 273-406.

Ready to submit your research? Choose BMC and benefit from:

- fast, convenient online submission

- thorough peer review by experienced researchers in your field

- rapid publication on acceptance

- support for research data, including large and complex data types

- gold Open Access which fosters wider collaboration and increased citations

- maximum visibility for your research: over $100 \mathrm{M}$ website views per year 